\title{
Compound optimization through data set-dependent chemical transformations
}

\author{
Antonio de la Vega de León ${ }^{*}$, Jürgen Bajorath \\ From 9th German Conference on Chemoinformatics \\ Fulda, Germany. 10-12 November 2013
}

Matched molecular pairs (MMPs) have previously been used to extract chemical transformations and study their effect on molecular properties such as activity [1,2]. Chemical transformations have been used to direct compound optimization efforts towards defined activity profiles [3]. Here we introduce a methodology to assess effects of chemical transformations based on MMPs of compounds active against specific targets. The effects of selected chemical transformations on drug design-relevant molecular properties were analyzed. For different data sets, transformations that were frequently found and induced favorable property changes were identified. These transformations were then iteratively applied to modify active compounds and move them into favorable regions of ADME-relevant property space. Activity of newly designed compounds was tracked using nearest-neighbor searches in ChEMBL. The results of our study indicate that activity-conservative data-set dependent transformation can aid in the design of new active compounds with favorable ADME characteristics [4].

Published: 11 March 2014

\section{References}

1. Wassermann AM, Bajorath J: Chemical substitutions that introduce activity cliffs across different compound classes and biological targets. J Chem Inf Model 2010, 50:1248-1256.

2. Wassermann AM, Bajorath J: Large-scale exploration of bioisosteric replacements on the basis of matched molecular pairs. Future Med Chem 2011, 3:425-436

3. Besnard J, Ruda GF, Setola V, Abecassis K, Rodriguiz RM, Huang X, Norval S, Sassano MF, Shin Al, Webster LA, Simeons FRC, Stojanovski L, Prat A, Seidah NG, Constam DB, Bickerton GR, Read KD, Wetsel WC, Gilbert IH, Roth BL, Hopkins AL: Automated Design of Ligands to Polypharmacological Profiles. Nature 2012, 492:215-220.

4. de León de la Vega A, Bajorath J: Compound Optimization through Data Set-Dependent Chemical Transformations. J Chem Inf Model 2013, 53:1263-1271.

\section{doi:10.1186/1758-2946-6-S1-P5}

Cite this article as: de León and Bajorath: Compound optimization through data set-dependent chemical transformations. Journal of Cheminformatics 2014 6(Suppl 1):P5. 